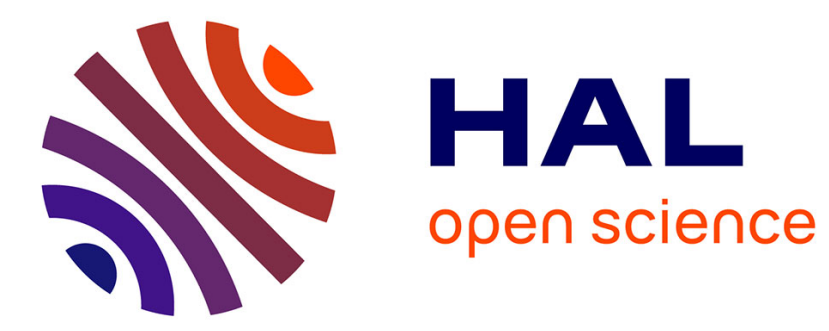

\title{
Equivalent stiffness and damping of sandwich piezoelectric beams submitted to active control
}

Laeticia Duigou, El Mostapha Daya, Michel Potier-Ferry

\section{To cite this version:}

Laeticia Duigou, El Mostapha Daya, Michel Potier-Ferry. Equivalent stiffness and damping of sandwich piezoelectric beams submitted to active control. Smart Materials and Structures, 2006, 15, pp.623-630. 10.1088/0964-1726/15/2/046 . hal-00400351

\section{HAL Id: hal-00400351 \\ https://hal.science/hal-00400351}

Submitted on 21 Jun 2018

HAL is a multi-disciplinary open access archive for the deposit and dissemination of scientific research documents, whether they are published or not. The documents may come from teaching and research institutions in France or abroad, or from public or private research centers.
L'archive ouverte pluridisciplinaire HAL, est destinée au dépôt et à la diffusion de documents scientifiques de niveau recherche, publiés ou non, émanant des établissements d'enseignement et de recherche français ou étrangers, des laboratoires publics ou privés. 


\title{
Equivalent stiffness and damping of sandwich piezoelectric beams submitted to active control
}

\author{
L Duigou $^{1}$, E M Daya ${ }^{2}$ and M Potier-Ferry ${ }^{2,3}$ \\ ${ }^{1}$ Laboratoire Génie Mécanique et Matériaux, Rue de Saint Maudé, BP 92116, 56321, \\ Lorient Cedex, France \\ ${ }^{2}$ Laboratoire de Physique et Mécanique des Matériaux, ISGMP, Université de Metz, \\ Ile du Saulcy, 57045 Metz, France \\ E-mail: laetitia.duigou@univ-ubs.fr and michel.potier-ferry@univ-metz.fr
}

In this paper, a modal approach is realized to define the damping properties of piezoelectric/elastic/piezoelectric beams. This method is based on the classical laminated beam theory and some simple assumptions about the electric fields. This leads to an electromechanical beam constitutive law. The piezoelectric layers play the role of sensor and actuator and two feedback control laws are considered. The equivalent stiffness, eigenfrequencies and loss factors of the whole system beam/control device are obtained.

\section{Introduction}

It is well known that piezoelectric materials can provide an effective way to reduce vibrations and noise. Thanks to this property, they are actually added to already existing structures in order to damp and control vibrations at low frequencies in many areas, such as in the aerospace industry. Thus, they can recover completely (layer) or partially (patch) the host structure. Many theoretical and experimental studies have been devoted to get accurate modelling and analysis of sandwich structures with piezoelectric layers such as beams or plates $[1,2,4,7,12,13]$. Also, the piezoelectric materials can be coupled with viscoelastic damped structures in order to yield hybrid active-passive damping. Indeed, the piezoelectric material permits damping the vibration modes at low frequency whereas the viscoelastic one yields to damping the modes at medium and high frequencies. So, a lot of hybrid (active-passive) damping configurations were developed in the literature to find the best solution which is able to decrease vibration amplitudes for a large number of modes. A review and assessment of these configurations can be found in [12].

From an engineering point of view, the damping properties can be defined by two modal quantities [3, 6, 11]: the loss factor (damping) and frequency. This approach of the

3 Author to whom any correspondence should be addressed. dynamical responses based on complex eigenfrequencies has been extensively applied in the case of viscoelastic damped sandwich structures and several numerical techniques have been proposed to characterize and to compute the complex eigenpairs [3, 11]. To our best knowledge, this simple modal approach has not yet been applied in the case of smart structures, involving piezoelectric laminates and active control.

In this paper, it is established that the modal approach can be useful in the case of elementary control laws. A piezoelectric/elastic/piezoelectric beam is studied. The piezoelectric layers play the role of sensor (s) and actuator (a) (see figure 1). Two feedback control laws are considered. A simple analytical study of this structure is carried out by using the classical laminate theory for the mechanical properties and by assuming a linearly varying electric field. Next, the electromechanical problem is reduced to a purely mechanical problem, which defines the elastic or viscoelastic equivalent stiffness properties. In the case of thin piezoelectric layers and of bending modes, some more simplifications can be introduced.

Another contribution of the present paper is the introduction of equivalent mechanical problems that include both the electromechanical behaviour of the thin piezoelectric layers and the control law. There are purely mechanical models in the literature, but only at a finite element level. Generally the electric quantities are condensed from an 


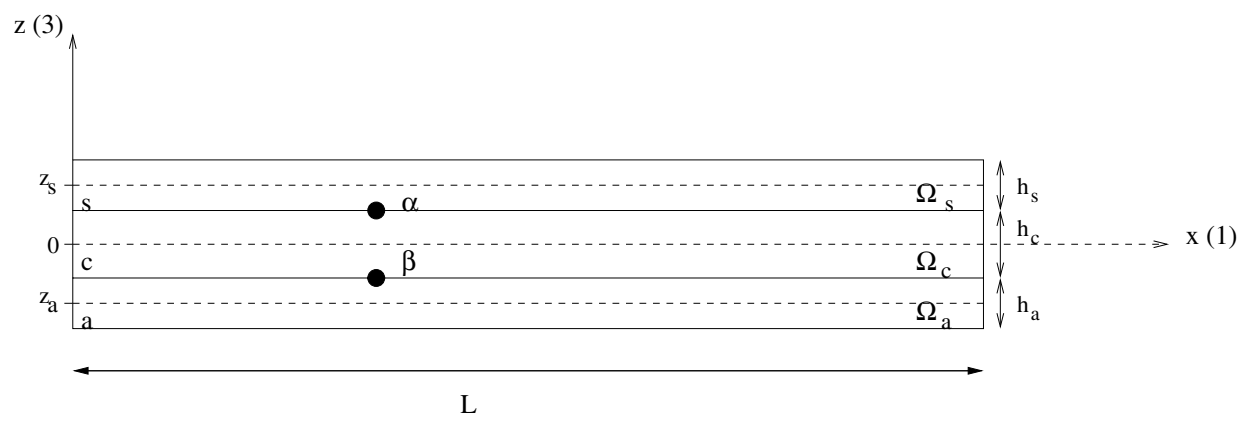

Figure 1. The piezoelectric/elastic/piezoelectric beam and notations.

electromechanical finite element model as in $[2,12], \ldots$. In [5], an analytical expression of the electric field is inserted into a finite element mechanical model. Here, we perform an analytical condensation of the electric field to get equivalent beam equations that are of elastic or viscoelastic type.

\section{An electromechanical model for piezoelectric laminated beams}

In this part, a model for a piezoelectric/elastic/piezoelectric beam is presented. In view of analytical studies, this model is designed to be as simple as possible including Euler-Bernoulli kinematics and the assumption of transverse electric fields. Various levels of approximations are discussed, as well as the treatment of the electrostatic problem in the sensor.

\subsection{Constitutive law}

Piezoelectric materials have the capacity to act, to react to an electric field or to a strain. This results in the following constitutive law:

$$
\begin{aligned}
& \sigma=\mathbf{c} \varepsilon-\mathbf{e}^{t} \mathbf{E} \\
& \mathbf{D}=\mathbf{e} \varepsilon+\epsilon \mathbf{E}
\end{aligned}
$$

where $\sigma, \varepsilon, \mathbf{D}$, and $\mathbf{E}$ are the stress tensor, strain tensor, electric displacement vector and electric field vector, respectively. c, e, and $\epsilon$ are the elasticity matrix, whose coefficients are measured at constant electric field, the piezoelectric matrix, and the dielectric permittivity tensor, whose components are measured at constant strain.

Orthotropic piezoelectric materials and an extension mechanism are considered. The constitutive relation can be represented in terms of a reduced number of components with respect to the three Cartesian/orthogonal axes, labelled 1, 2 and 3 of piezoelectric material. We suppose that the piezoelectric layers are thin, the stress tensor is uniaxial and the electric fields $\mathbf{D}$ and $\mathbf{E}$ parallel to the transverse direction. So, the constitutive law is reduced to [2]

$$
\begin{gathered}
\left\{\begin{array}{l}
\sigma_{1} \\
D_{3}
\end{array}\right\}=\left[\begin{array}{cc}
c_{11}^{*} & -e_{31}^{*} \\
e_{31}^{*} & \epsilon_{33}^{*}
\end{array}\right]\left\{\begin{array}{l}
\varepsilon_{1} \\
E_{3}
\end{array}\right\} \\
\epsilon_{33}^{*}=\epsilon_{33}+\frac{e_{33}^{2}}{c_{33}} \\
e_{31}^{*}=e_{31}-\frac{c_{13}}{c_{33}} e_{33} \\
c_{11}^{*}=c_{11}-\frac{c_{13}^{2}}{c_{33}}
\end{gathered}
$$

\subsection{Electric description}

The electric field-electric potential relation is the following:

$$
E_{3}=-\frac{\partial \phi}{\partial z} .
$$

We consider that the central layer is conductive with an uniform potential, fixed to zero [7]. Often in the literature, the potential field is assumed to be linearly varying across the thickness, in accordance with the same variation of the stress and strain [8-10]. It may be asked whether a constant electric field gives a sufficient description in the case of a thin layer. An adimensional variable $r(0 \leqslant r \leqslant 1)$ is introduced in piezoelectric layers, for instance by $z=\frac{h_{c}}{2}+h_{s} r$ in the top layer. So, the electric field is written in terms of two unknowns $\phi_{1}$ and $\phi_{2}$ :

$$
E_{3}=-\frac{1}{h_{s}}\left(\phi_{1}+\phi_{2}(1-2 r)\right)
$$

where $\phi_{1}=\phi_{1}(x)$ represents the superficial potential and $\phi_{2}=\phi_{2}(x)$ the internal potential variation.

\subsection{Kinematics}

To describe the properties of a sandwich piezoelectric/elastic/piezoelectric beam, we use the classical theory of laminates. The kinematics of the structure is represented by the classical hypothesis of Euler-Bernoulli:

$$
\begin{gathered}
u(x, z)=u(x)-z w^{\prime}(x) \\
w(x, z)=w(x) \\
\varepsilon_{1}=u^{\prime}-z w^{\prime \prime} .
\end{gathered}
$$

\subsection{Electromechanical law of the sandwich beam}

Now a global electromechanical behaviour law is established. In this respect, the definitions of the normal force $N_{i}$ and the bending moment $M_{i}$ of each layer are needed:

$$
\begin{gathered}
N_{i}=\int_{S_{i}} \sigma_{i} \mathrm{~d} S \text { and } M_{i}=\int_{S_{i}}\left(z-z_{i}\right) \sigma_{i} \mathrm{~d} S \\
\quad i=s, c, a .
\end{gathered}
$$

Because of the constitutive law (2), the uniaxial stress $\sigma_{1 i}$ is equal to

$$
\begin{aligned}
& \sigma_{1 c}=E_{c}\left(u^{\prime}-z w^{\prime \prime}\right) \quad \text { for the central elastic layer } \\
& \sigma_{1 i}=c_{11}^{*}\left(u^{\prime}-\left(z-z_{i}\right) w^{\prime \prime}\right)-e_{31}^{*} E_{3}
\end{aligned}
$$


In accordance with Euler-Bernoulli kinematics, the beam stresses can be represented by a global normal force $N$ and a global bending moment $M$. Classically, they are expressed as functions of the normal forces and bending moments of each layer in the following form:

$$
\begin{gathered}
N=N_{s}+N_{c}+N_{a} \\
M=M_{s}+M_{c}+M_{a}-z_{s} N_{s}-z_{a} N_{a}
\end{gathered}
$$

with $z_{s}=\frac{h_{c}+h_{s}}{2}$ and $z_{a}=-\frac{h_{c}+h_{a}}{2}$.

By combining (6) and (7), the global electromechanical constitutive law of the laminate is deduced:

$$
\begin{aligned}
N= & (E S)_{\mathrm{mec}} u^{\prime}+B_{\mathrm{mec}} w^{\prime \prime}+e_{31}^{*}\left(\frac{S_{s}}{h_{s}} \phi_{1 s}-\frac{S_{a}}{h_{a}} \phi_{1 a}\right) \\
M= & B_{\mathrm{mec}} u^{\prime}+(E I)_{\mathrm{mec}} w^{\prime \prime}-e_{31}^{*}\left(\frac{z_{s} S_{s}}{h_{s}} \phi_{1 s}-\frac{z_{a} S_{a}}{h_{a}} \phi_{1 a}\right) \\
& -\frac{e_{31}^{*}}{6}\left(S_{s} \phi_{2 s}-S_{a} \phi_{2 a}\right)
\end{aligned}
$$

with

$$
\begin{gathered}
(E S)_{\mathrm{mec}}=E_{c} S_{c}+c_{11}^{*}\left(S_{s}+S_{a}\right) \\
B_{\mathrm{mec}}=-c_{11}^{*}\left(z_{s} S_{s}+z_{a} S_{a}\right) \\
(E I)_{\mathrm{mec}}=E_{c} I_{c}+c_{11}^{*}\left(I_{s}+I_{a}\right)+c_{11}^{*}\left(S_{s} z_{s}^{2}+S_{a} z_{a}^{2}\right) .
\end{gathered}
$$

So (8) is the global constitutive law, that expresses the beam generalized stresses $N$ and $M$ as functions of the membrane strain $u^{\prime}$, of the curvature $w^{\prime \prime}$, of the superficial potentials $\phi_{1 s}$ and $\phi_{1 a}$ and on two additional quantities $\phi_{2 s}$ and $\phi_{2 a}$ for the internal variations of the electric field. A few remarks can be made about this constitutive law. First, without electric fields, one recognizes the classical laminated beam theory. Then, $B_{\text {mec }}$ represents the membrane/bending coupling term. If the beam is symmetrical $\left(z_{a}=-z_{s}\right), B_{\mathrm{mec}}$ is equal to zero, that is to say there is no membrane/bending coupling. Finally, the electric quantities $\phi_{1 a}, \phi_{2 a}, \phi_{1 s}$ and $\phi_{2 s}$ can be considered as forcing terms of a classical beam model.

\subsection{Condensation of electric terms in sensor layer}

With the help of the Galerkin method, the superficial potential and internal potential variation of the sensor can be written as functions of strain quantities $u^{\prime}$ and $w^{\prime \prime}$ (see the appendix):

$$
\begin{gathered}
\phi_{s}=\phi_{1 s} r+\phi_{2 s} r(1-r) \\
\phi_{1 s}=\frac{e_{31}^{*}}{\epsilon_{33}^{*}} h_{s}\left(u^{\prime}-z_{s} w^{\prime \prime}\right) \\
\phi_{2 s}=\frac{e_{31}^{*}}{2 \epsilon_{33}^{*}} h_{s}^{2} w^{\prime \prime} .
\end{gathered}
$$

Next, we replace $\phi_{1 s}$ and $\phi_{2 s}$ by their expression (10) in (8). So, we obtain a new constitutive law, which takes account of electric boundary conditions in the sensor:

$$
\begin{gathered}
N=(E S)_{\mathrm{eq}} u^{\prime}+B_{\mathrm{eq}} w^{\prime \prime}-e_{31}^{*} \frac{S_{a}}{h_{a}} \phi_{1 a} \\
M=B_{\mathrm{eq}} u^{\prime}+(E I)_{\mathrm{eq}} w^{\prime \prime}+e_{31}^{*} S_{a}\left(\frac{z_{a}}{h_{a}} \phi_{1 a}+\frac{1}{12} \phi_{2 a}\right)
\end{gathered}
$$

with

$$
\begin{gathered}
(E S)_{\mathrm{eq}}=(E S)_{\mathrm{mec}}+\frac{e_{31}^{* 2}}{\epsilon_{33}^{*}} S_{s} \\
B_{\mathrm{eq}}=B_{\mathrm{mec}}-\frac{e_{31}^{* 2}}{\epsilon_{33}^{*}} z_{s} S_{s} \\
(E I)_{\mathrm{eq}}=(E I)_{\mathrm{mec}}+\frac{e_{31}^{* 2}}{\epsilon_{33}^{*}} S_{s}\left(z_{s}^{2}-\frac{h_{s}^{2}}{12}\right) .
\end{gathered}
$$

So the electric behaviour of the sensor modifies the apparent stiffness of the beam, by increasing the membrane stiffness $E S$ and the bending stiffness $E I$. Considering the expression of $B_{\mathrm{eq}}$, one sees that this electric condensation induces a membrane bending coupling, even with a symmetric beam.

This dissymmetry of the laminate behaviour does not arise from a material dissymmetry, but from the difference of electric boundary conditions between the sensor and the actuator.

\subsection{What approximations are needed in the case of thin piezoelectric layers?}

To simplify this discussion, let us consider a symmetrical beam:

$$
h_{a}=h_{s}, \quad S_{a}=S_{s}, \quad z_{a}=-z_{s} .
$$

Then, the term $B_{\text {mec }}$ in the constitutive law becomes zero and the others are rewritten in the following form:

$$
\begin{gathered}
(E S)_{\mathrm{eq}}=E_{c} S_{c}+2 c_{11}^{*} S_{s}+\frac{e_{31}^{* 2}}{\epsilon_{33}^{*}} S_{s} \\
B_{\mathrm{eq}}=-\frac{e_{31}^{* 2}}{\epsilon_{33}^{*}} z_{s} S_{s} \\
(E I)_{\mathrm{eq}}=E_{c} I_{c}+2 c_{11}^{*} I_{s}+2 c_{11}^{*} S_{s} z_{s}^{2}+\frac{e_{31}^{* 2}}{\epsilon_{33}^{*}} S_{s}\left(z_{s}^{2}-\frac{h_{s}^{2}}{12}\right) .
\end{gathered}
$$

By comparison with the one-layer beam, we want to evaluate the extra terms due to the direct effect of the piezoelectric layers or those due to the electrostatic condensation in the sensor. To simplify, a rectangular symmetric cross-section is considered. This leads to

$$
\begin{aligned}
I_{j} & \rightarrow \frac{h_{j}^{3}}{12} \\
S_{j} & \rightarrow h_{j} .
\end{aligned}
$$

Then, three adimensional parameters are introduced:

$$
r_{E}=\frac{c_{11}^{*}}{E_{c}}, \quad r_{E}^{p}=\frac{e_{31}^{* 2}}{c_{11}^{*} \epsilon_{33}^{*}}, \quad r_{h}=\frac{h_{s}}{h_{c}}
$$

where $r_{E}$ and $r_{E}^{p}$ represent material ratios and $r_{h}$ a thickness ratio.

First, the second term of $(E S)_{\mathrm{eq}}$ represents the direct contribution of the piezoelectric layers to the stiffness, and the last term the effect of the electrostatic condensation. Using the hypotheses (15) and (16), the following equivalences can be written:

$$
\begin{gathered}
\frac{2 c_{11}^{*} S_{s}}{E_{c} S_{c}} \simeq 2 r_{E} r_{h} \\
\left(\frac{e_{31}^{* 2}}{\epsilon_{33}^{*}} S_{s}\right) /\left(2 c_{11}^{*} S_{s}\right)=r_{E}^{p} / 2 .
\end{gathered}
$$


In order to evaluate the adimensional terms, let us consider the sandwich beam studied by Zhang and Sun [13]. It is constituted by PZT5H material in the skins and aluminium in the core. Its geometric characteristics are $L=10 \mathrm{~cm}, h_{c}=1.6 \mathrm{~cm}$ and $h_{s}=h_{a}=0.1 \mathrm{~cm}$. With these data, the approximated values of the adimensional ratios are

$$
r_{E} \simeq 1, \quad r_{E}^{p} \simeq 0.5, \quad r_{h} \simeq 6 \times 10^{-2} .
$$

The material ratios $\left(r_{E}, r_{E}^{p}\right)$ are neither small nor big, whereas the thickness ratio is sufficiently little. Therefore, the second and third terms of $(E S)_{\text {eq }}$ represent respectively $12 \%$ and 3\% of the first one. So, they cannot be neglected.

The same study is realized for $(E I)_{\mathrm{eq}}$. In this case, the terms due to the direct effect of piezoelectric layers $2 c_{11}^{*}\left(I_{s}+\right.$ $S_{s} z_{s}^{2}$ ) and the term due to the electrostatic condensation $\frac{e_{31}^{* 2}}{\epsilon_{33}^{*}} S_{s}\left(z_{s}^{2}-\frac{h_{s}^{2}}{12}\right)$ are compared to the bending stiffness $E_{c} I_{c}$ of the central layer:

$$
\begin{gathered}
\frac{2 c_{11}^{*}\left(I_{s}+S_{s} z_{s}^{2}\right)}{E_{c} I_{c}}=2 r_{E}\left(r_{h}^{3}+3 r_{h}\left(1+r_{h}\right)^{2}\right) \\
\frac{e_{31}^{* 2}}{\epsilon_{33}^{*} E_{c} I_{c}} S_{s}\left(z_{s}^{2}-\frac{h_{s}^{2}}{12}\right)=r_{E}^{p} r_{E} r_{h}\left(3\left(1+r_{h}\right)^{2}-r_{h}^{2}\right) .
\end{gathered}
$$

Considering again the sandwich beam studied by Zhang and Sun, these terms are respectively about $36 \%$ and $10 \%$. But, they can be simplified by neglecting the terms involving $r_{h}^{3}$ and even $r_{h}^{2}$. Two terms are functions of $r_{h}^{3}$ : the bending stiffness of the piezoelectric layers $2 r_{E} r_{h}^{3}$ and the quadratic term of the potential $-r_{E}^{p} r_{E} r_{h}^{3}$. These last terms represent respectively $0.04 \%$ and $0.01 \%$ of $E_{c} I_{c}$. Therefore, two more assumptions can be introduced to get a simpler model. First, the stress in the skins can be reduced to the membrane contribution (i.e., the bending moments $M_{s}$ and $M_{a}$ can be neglected). Second, the quadratic term $\phi_{2 s}$ in the electric potential has no influence on the global behaviour and can be dropped. This means that the assumption of constant electric fields is relevant for thin skins $\left(r_{h}\right.$ lower than 0.2$)$. In what follows, we limit ourselves to this simplified analysis.

\section{A simplified analysis for bending modes}

In this part, a new simplification is introduced to analyse the bending modes of symmetrical sandwich beams. We assume further:

$$
u \approx 0 \quad \text { and } \quad\left|u^{\prime}\right| \ll z_{s}\left|w^{\prime \prime}\right| .
$$

This means that the membrane/bending coupling is neglected and that the bending constitutive law is reduced to

$$
M=(E I)_{\mathrm{eq}} w^{\prime \prime}-e_{31}^{*} S_{s} \frac{z_{s}}{h_{s}} \phi_{1 a}
$$

with $(E I)_{\mathrm{eq}}$ defined in (14). Let us recall that $\phi_{2 a}$ has been dropped according the argument given in section 2.6.

At this level, feedback control laws will be introduced, by connecting the two potentials in the piezoelectric layers. These control laws will be condensed, to get a global bending law, including the three layers of the laminate and the control device.

\subsection{Direct proportional feedback control}

With this control law, the superficial potential applied to the actuator is directly proportional to the superficial potential of the sensor:

$$
\phi_{1 a}(x)=G_{c} \phi_{1 s}(x)
$$

where $G_{c}$ represents the control gain.

According to the assumptions (20) and $\phi_{2 a}=0$ and to the law (22), the superficial potential becomes

$$
\phi_{1 a}=-G_{c} \frac{e_{31}^{*}}{\epsilon_{33}^{*}} h_{s} z_{s} w^{\prime \prime}
$$

By combining (21) and (23), a sort of equivalent elastic constitutive law for the whole system (beam and control device) is obtained. This yields a classical form for the elastic bending of beams, involving a simplified expression of the system bending stiffness $(E I)_{\text {simp }}$ :

$$
\begin{gathered}
M=(E I)_{\mathrm{simp}} w^{\prime \prime} \\
(E I)_{\text {simp }}=(E I)_{\mathrm{eq}}+G_{c} S_{s} \frac{e_{31}^{* 2}}{\epsilon_{33}^{*}} z_{s}^{2} .
\end{gathered}
$$

Therefore, a symmetrical piezoelectric/elastic/piezoelectric beam can be represented by an equivalent homogeneous beam, whose stiffness matrix increases with the control gain $G_{c}$. One remarks that the increase of stiffness depends on the following parameter:

$$
g=G_{c} S_{s} \frac{e_{31}^{* 2}}{\epsilon_{33}^{*}}
$$

that is a function of the control gain and of the dielectric and piezoelectric constants. It could be also convenient to consider a non-dimensional constant $r_{\text {control }}$ as follows:

$$
r_{\text {control }}=\frac{G_{c} S_{s}}{E_{c} I_{c}} \frac{e_{31}^{* 2}}{\epsilon_{33}^{*}} Z_{s}^{2}=\frac{g Z_{s}^{2}}{E_{c} I_{c}} .
$$

So the simplified system bending stiffness can be rewritten in a more convenient form, where $(E I)_{\mathrm{eq}}$ has been defined in (12) and (14):

$$
(E I)_{\mathrm{simp}}=(E I)_{\mathrm{eq}}+g Z_{s}^{2}=\left(E_{c} I_{c}\right)\left[\frac{(E I)_{\mathrm{eq}}}{E_{c} I_{c}}+r_{\text {control }}\right] .
$$

The balance of momentum leads to the following equation:

$$
M^{\prime \prime}+(\rho S)_{\mathrm{eq}} \ddot{w}=0
$$

where $(\rho S)_{\mathrm{eq}}$ is the equivalent linear density of the sandwich beam:

$$
(\rho S)_{\mathrm{eq}}=\rho_{s} S_{s}+\rho_{c} S_{c}+\rho_{a} S_{a} .
$$

The modal solutions of (24) and (27) are classical [6], and the $n$th eigenfrequency is given by

$$
\omega_{n}^{2}=\left(\mu_{n}\right)^{4} \frac{(E I)_{\mathrm{simp}}}{(\rho S)_{\mathrm{eq}} L^{4}}
$$

where the non-dimensional numbers $\mu_{n}$ depend on the boundary conditions. For instance, for a bi-articulated beam, $\mu_{n}=n \pi$.

So the control law (22) leads only to an increase of the global stiffness of the beam (if $G_{c}$ is positive) and therefore of 
the eigenfrequencies, that are given by (24), (28), and (29), for any boundary condition. Such a law does not induce damping, because the eigenfrequencies (29) are real.

This increase of stiffness can be characterized by the parameter $g$ (see (25)) or by the adimensional parameter $r_{\text {control }}$, which is a function of the control gain and of the material and geometrical parameters of the laminate.

\subsection{Direct and proportional velocity feedback control}

In the case of the direct and proportional velocity feedback control, the potential applied to the actuator is proportional to the time derivative of the sensor potential:

$$
\phi_{a}(x)=G_{c} \dot{\phi}_{s}(x)
$$

with $G_{c}$ positive. As previously, the electric field is assumed to be constant in each piezoelectric layer. Using (10), (20) and considering $\phi_{2 s}=0$ (see section 2.6), equation (30) leads to

$$
\phi_{1 a}=-G_{c} \frac{e_{31}^{*}}{\epsilon_{33}^{*}} h_{s} z_{s} \dot{w}^{\prime \prime} .
$$

As before, this $\phi_{1 a}$ is replaced in the expression of bending moment (21) and we obtain

$$
M=(E I)_{\mathrm{eq}} w^{\prime \prime}+g z_{s}^{2} \dot{w}^{\prime \prime}
$$

where the parameter $g$ represents the term of control and is defined as previously (25). This relation (32) is a sort of viscoelastic bending law. The viscoelasticity is of Kelvin type and it is due only to the control law (30).

Considering a problem of free vibrations, the deflection $w(x)$ can be sought in the form $w=W(x) \mathrm{e}^{\mathrm{i} \omega t}$ and the constitutive law (32) is rewritten in the following form:

$$
M=\left[(E I)_{\mathrm{eq}}+\mathrm{i} \omega g z_{s}^{2}\right] W^{\prime \prime} .
$$

Therefore, $M$ is defined like $M=E I(\omega) w^{\prime \prime}$. This corresponds to a complex stiffness matrix linearly dependent on $\omega$. So the behaviour of the whole system is equivalent to that of a viscoelastic beam and a loss factor can be defined for each vibration mode. Using the equivalent constitutive law (32), the dynamic equation (27) leads to

$$
W^{(4)}-(\rho S)_{\mathrm{eq}} \frac{\omega^{2}}{E I(\omega)} W=0 .
$$

From Geradin [6], the ratio $L^{4}\left(\rho_{S}\right)_{\mathrm{eq}} \omega^{2} / E I(\omega)$ is real and depends only on the boundary conditions and on the mode. These values are exactly the adimensional numbers $\mu_{n}$, recalled previously. Because $E I(\omega)$ is complex, the solution $\omega$ of the free vibration problem is complex and a damping can be defined by the following equation in $\omega$ :

$$
\omega_{n}^{2}=\frac{\mu_{n}^{4}}{\left(\rho_{S}\right)_{\mathrm{eq}}}\left[(E I)_{\mathrm{eq}}+\mathrm{i} \omega g z_{s}^{2}\right]
$$

To simplify, the eigenfrequency of a beam only constituted by the elastic core is introduced as a reference:

$$
\omega_{\mathrm{nc}}^{2}=\mu_{n}^{4} \frac{E_{c} I_{c}}{\rho_{c} S_{c} L^{4}} .
$$

So, equation (35) becomes

$$
\begin{gathered}
\lambda_{n}^{2}-\mathrm{i} \lambda_{n} \beta_{n}-1=0 \\
\lambda_{n}=\frac{\omega}{\omega_{\mathrm{nc}}} \\
\beta_{n}=g \frac{z_{s}^{2}}{E_{c} I_{c}} \omega_{\mathrm{nc}}=r_{\text {control }} \omega_{\mathrm{nc}}
\end{gathered}
$$

where $r_{\text {control }}$ is defined as in (26). Note that, in the present case, $r_{\text {control }}$ is not adimensional, because of a different definition of the control gain.

The solution of this equation is

$$
\lambda_{n}=\frac{\mathrm{i} \beta_{n} \pm \overline{4-\beta_{n}^{2}}}{2} .
$$

The damping $\eta_{n}$ is defined classically by $\omega_{n}^{2}=\Omega_{n}^{2}\left(1+\mathrm{i} \eta_{n}\right)$, where $\Omega_{n}$ represents the real frequency. Here, it is equal to

$$
\eta_{n}=\frac{\beta_{n} \overline{4-\beta_{n}^{2}}}{2-\beta_{n}^{2}}
$$

with $\beta_{n}$ defined in (37).

If $\beta_{n} \ll 1$, the damping is about $\beta_{n}$; that is to say,

$$
\eta_{n}=G_{c} \omega_{\mathrm{nc}} \frac{S_{s} z_{s}^{2}}{I_{c}} \frac{e_{31}^{* 2}}{E_{c} \epsilon_{33}^{*}}=r_{\text {control }} \omega_{\mathrm{nc}} .
$$

In any case, each modal damping $\eta_{n}$ depends only on a adimensional parameter $\beta_{n}=r_{\text {control }} \omega_{\text {nc }}$, a function of a parameter $r_{\text {control }}$ similar to that given above and of the reference frequency $\omega_{\mathrm{nc}}$. This second type of control law induces a damping that is proportional to $G_{c}$, but does not modify the eigenfrequency very much.

\subsection{Are the problems well posed?}

With the first control law (22), the resulting equations (24), (27) are those of an elastic beam. This equation is known to be well posed, at least if the equivalent modulus $(E I)_{\text {simp }}$ is positive. So the gain can be positive or negative and not too large. The well posed condition is

$$
r_{\text {control }} \geqslant-\frac{(E I)_{\mathrm{eq}}}{E_{c} I_{c}} .
$$

With the control law (30), one gets the equations of a Kelvin viscoelastic beam (27), (32), that are well posed only when the gain is not negative.

These equivalent mechanical models have never been presented in the literature. This result has consequences on the relevant discretization techniques. For instance, the control term in (32) leads to a parabolic equation so that the time derivative must be discretized in an implicit way to get a stable scheme.

\section{A second analysis taking into account the axial terms}

In this last part, we study the bending modes of a sandwich beam with active control but without neglecting the axial strain and axial inertia term as in the previous section. This 
will permit us to define the limits of this simplified analysis. Nevertheless, the assumption of a constant electric field per layer $\left(\phi_{2 s}=\phi_{2 a}=0\right)$ will be kept, because it has been established in section 2. Within beam theory, the balance of momentum leads to

$$
\begin{aligned}
-N^{\prime}+(\rho S)_{\mathrm{eq}} \ddot{u} & =0 \\
M^{\prime \prime}+(\rho S)_{\mathrm{eq}} \ddot{w} & =0
\end{aligned}
$$

where $M$ and $N$ are defined by the constitutive laws (11), (14) and (15) and $(\rho S)_{\text {eq }}$ by (28).

The two previous control laws will be considered again. For simplicity, the analysis is limited to a simply support beam $(N=M=w=0$ at $x=0, L)$.

\subsection{Direct proportional feedback control}

With the direct proportional feedback control law, the equivalent constitutive law of the system is defined from (8), (22) and (10):

$$
\left\{\begin{array}{l}
N \\
M
\end{array}\right\}=\left[\begin{array}{cc}
(E S)_{\mathrm{eq}}-g & B_{\mathrm{eq}}+g z_{s} \\
B_{\mathrm{eq}}-g z_{s} & (E I)_{\mathrm{eq}}+g z_{s}^{2}
\end{array}\right]\left\{\begin{array}{c}
u^{\prime} \\
w^{\prime \prime}
\end{array}\right\}
$$

where $g$ represents the term of control and is defined by (25).

As compared with (24), this constitutive law of the system couples membrane and bending quantities. The constitutive matrix depends on the control parameter $g$. Note that it is not symmetric, which is due to the non-conservative character of the control law.

The solution of the system (42), (43) is sought in the following form:

$$
\begin{aligned}
& u=q_{1} \cos \left(\frac{n \pi x}{L}\right) \mathrm{e}^{\mathrm{i} \omega t} \\
& w=q_{2} \sin \left(\frac{n \pi x}{L}\right) \mathrm{e}^{\mathrm{i} \omega t} .
\end{aligned}
$$

4.1.1. Effect of membrane/bending coupling. To study the effect of membrane/bending coupling, a membrane static condensation is considered in the first equation of (42). Thus, $q_{1}$ can be expressed according to $q_{2}$ and it is replaced in the second equation of (42). This permits us to obtain the bending frequency, which is equal to

$$
\left.\omega_{n}^{2}=\frac{n^{4} \pi^{4}}{(\rho S)_{\mathrm{eq}} L^{4}} \frac{-\left(B_{\mathrm{eq}}^{2}-g^{2} z_{s}^{2}\right)}{(E S)_{\mathrm{eq}}-g}+(E I)_{\mathrm{eq}}+g z_{s}^{2}\right] .
$$

As in the previous study, with this law, the frequency is real and there is no damping. Compared to (29), $\omega_{n}^{2}$ of (45) includes an extra term because of taking into account the membrane motion. This term is compared to the bending stiffness $\left(E_{c} I_{c}\right)$ of the central layer:

$$
\begin{aligned}
& \frac{-\left(B_{\mathrm{eq}}^{2}-g^{2} z_{s}^{2}\right)}{\left[(E S)_{\mathrm{eq}}-g\right] E_{c} I_{c}}=-\frac{3 r_{E}^{2} r_{E}^{p 2} r_{h}^{2}\left(1+r_{h}\right)^{2}\left(1-G_{c}^{2}\right)}{1+2 r_{E} r_{h}+r_{E} r_{E}^{p} r_{h}\left(1-G_{c}\right)} \\
& =3 \times 10^{-3} \frac{G_{c}^{2}-1}{1-G_{c} / 38} .
\end{aligned}
$$

The last equality holds with the numerical values presented in section 2. Hence the influence of the axial strain is small $(<0.3 \%)$ for a gain lower than 1 . From (46) and figure 2, it appears that this coupling term can be neglected for $G_{c}$ lower than 3 .

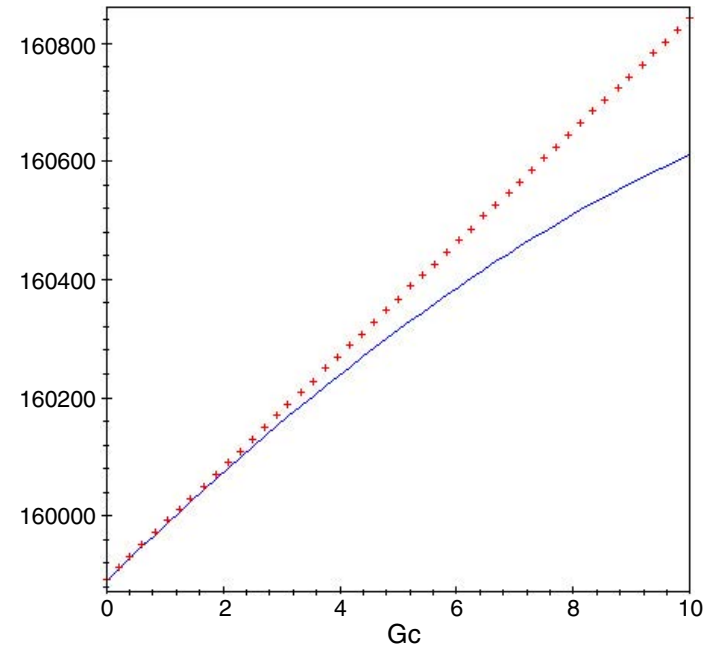

Figure 2. Modal frequency $\omega_{n}$ as function of the control gain constant $G_{c}$. Simply supported beam. Study of the first vibration mode. Direct proportional feedback control. $(x)$ results from formula (29), (continuous line) results from formula (45).

4.1.2. Effect of the membrane inertia. Here equations (42) and (43) are solved without any simplification. This yields, by letting $k=n \pi / L$,

$$
\begin{array}{cc}
{\left[(E S)_{\mathrm{eq}}-g\right] k^{2}-(\rho S)_{\mathrm{eq}} \omega^{2}} & \left(B_{\mathrm{eq}}+g z_{s}\right) k^{3} \\
\left(B_{\mathrm{eq}}-g z_{s}\right) k^{3} & {\left[(E I)_{\mathrm{eq}}+g z_{s}^{2}\right] k^{4}-(\rho S)_{\mathrm{eq}} \omega^{2}} \\
\times \quad \begin{array}{l}
q_{1} \\
q_{2}=0
\end{array} . &
\end{array}
$$

Thus, the eigenfrequencies are given by an equation of the second degree with $(\rho S)_{\mathrm{eq}} \omega^{2}$ as unknown. Here we only discuss in which conditions the axial inertia term is small. So it is sufficient to compare the first and the last term of the $(1,1)$ term of the matrix (47). Because only an order of magnitude is sought, we suppose

$$
\omega=\omega_{\mathrm{nc}}, \quad(\rho S)_{\mathrm{eq}}=\rho_{c} S_{c}, \quad(E S)_{\mathrm{eq}}=E_{c} S_{c} .
$$

Also, the expression (36) of $\omega_{\mathrm{nc}}$ and hypothesis (15) are considered. Then, the ratio $(\rho S)_{\mathrm{eq}} \omega^{2} /(E S)_{\mathrm{eq}} k^{2}$ becomes

$$
\frac{(\rho S)_{\mathrm{eq}} \omega^{2}}{(E S)_{\mathrm{eq}} k^{2}} \simeq n^{2} \pi^{2} \frac{I_{c}}{L^{2} S_{c}} \simeq \frac{n^{2} \pi^{2}}{12}\left(\frac{h_{c}}{L}\right)^{2} .
$$

This ratio depends on the mode $\left(n^{2}\right)$ and on the beam slenderness $\left(h_{c} / L\right)$. So, the effect of the membrane inertia can be neglected for an aspect ratio $L / n h_{c}$ greater than 10 .

\subsection{Direct and proportional velocity feedback control}

In this case, the two potentials $\phi_{a}$ and $\phi_{s}$ are related by the law (30). Because of (10), the constitutive law of the whole system is then expressed in the following form:

$$
\left\{\begin{array}{l}
N \\
M
\end{array}\right\}=\left[\begin{array}{cc}
(E S)_{\mathrm{eq}} & B_{\mathrm{eq}} \\
B_{\mathrm{eq}} & (E I)_{\mathrm{eq}}
\end{array}\right]\left\{\begin{array}{c}
u^{\prime} \\
w^{\prime \prime}
\end{array}\right\}+\left[\begin{array}{cc}
-g & g z_{s} \\
-g z_{s} & g z_{s}^{2}
\end{array}\right]\left\{\begin{array}{c}
\dot{u}^{\prime} \\
\dot{w}^{\prime \prime}
\end{array}\right\}
$$

where $g$, always given by (25), represents the control term Once more, this global law is viscoelastic of Kelvin type. Note that the damping matrix is not symmetric. 
The analysis of the vibration modes of a bi-articulated beam is similar to that in 4.1 .

Here, we limit our approach by using a static approximation in the axial equation. Then, the second equation of system (42) is written as

$$
\begin{array}{r}
-(\rho S)_{\mathrm{eq}} \omega^{2}+\mathrm{i} \omega g z_{s} \frac{n^{4} \pi^{4}}{L^{4}}\left(\frac{B_{\mathrm{eq}}}{(E S)_{\mathrm{eq}}}+z_{s}\right) \\
+\frac{n^{4} \pi^{4}}{L^{4}}\left(-\frac{B_{\mathrm{eq}}^{2}}{(E S)_{\mathrm{eq}}}+(E I)_{\mathrm{eq}}\right)=0 .
\end{array}
$$

The solution $\omega^{2}$ of this last equation is complex. That is to say, a damping can be defined. Compared to (34), equation (51) includes two extra terms $\left(\frac{B_{\mathrm{eq}}}{(E S)_{\mathrm{eq}}}\right)$ and $\left(-\frac{B_{\mathrm{eq}}^{2}}{(E S)_{\mathrm{eq}}}\right)$ due to the membrane strain. To obtain the damping, equation (51) must be solved:

$$
\begin{gathered}
\lambda_{n}^{2}-\mathrm{i} \lambda_{n} \beta_{n}-\gamma=0 \\
\lambda_{n}=\frac{\omega}{\omega_{\mathrm{nc}}} \\
\beta_{n}=G_{c} \omega_{\mathrm{nc}} \frac{e_{31}^{* 2}}{E_{c} \epsilon_{33}^{*}} \frac{S_{s} z_{s}}{I_{c}}\left(\frac{B_{\mathrm{eq}}}{E_{c} S_{c}}+z_{s}\right) \\
\gamma=1-\frac{B_{\mathrm{eq}}^{2}}{E_{c}^{2} S_{c} I_{c}} .
\end{gathered}
$$

The solution of equation (52) is

$$
\lambda_{n}=\frac{\mathrm{i} \beta_{n} \pm \sqrt{4 \gamma-\beta_{n}^{2}}}{2} .
$$

The damping is defined as previously, and is equal to

$$
\eta_{n}=\frac{\beta_{n} \overline{4 \gamma-\beta_{n}^{2}}}{2 \gamma-\beta_{n}^{2}} .
$$

If $\beta_{n} \ll 1$ and $\gamma \simeq 1$, the damping is about $\beta_{n}$; that is to say,

$$
\eta_{n} \simeq \beta_{n}=3 G_{c} \omega_{\mathrm{nc}} r_{h} r_{E} r_{E}^{p}\left(1+r_{h}\right)^{2}\left(1-r_{h} r_{E} r_{E}^{p}\right) .
$$

With the numerical data (18) for $r_{E}$ and $r_{E}^{p}$, it becomes

$$
\eta_{n} \simeq \frac{3}{2} G_{c} \omega_{\mathrm{nc}} r_{h}\left(1+r_{h}\right)^{2}\left(1-\frac{r_{h}}{2}\right) .
$$

In the case of thin piezoelectric layers $\left(r_{h} \ll 1\right.$, then $\beta_{n} \ll 1$ and $\gamma \simeq 1$ ) the damping (56) is consistent with the values (40) that had been found in the previous section. This establishes than the membrane strains can be neglected for thin skins. Indeed, these comments are in good agreement with the results presented in figure 3. In this, the results from formula (39) and those from (54) are reported for comparison.

\subsection{Are the problems well posed?}

It is not so obvious to decide if the equations (42), (43) lead to a well posed problem, because the stiffness matrix in (43) is not symmetric. Nevertheless the elastic term does not satisfy the ellipticity conditions if the gain $g$ is sufficiently large and especially when

$$
g>(E S)_{\mathrm{eq}} \quad \text { or } \quad g<-\frac{(E I)_{\mathrm{eq}}}{z_{s}^{2}} .
$$

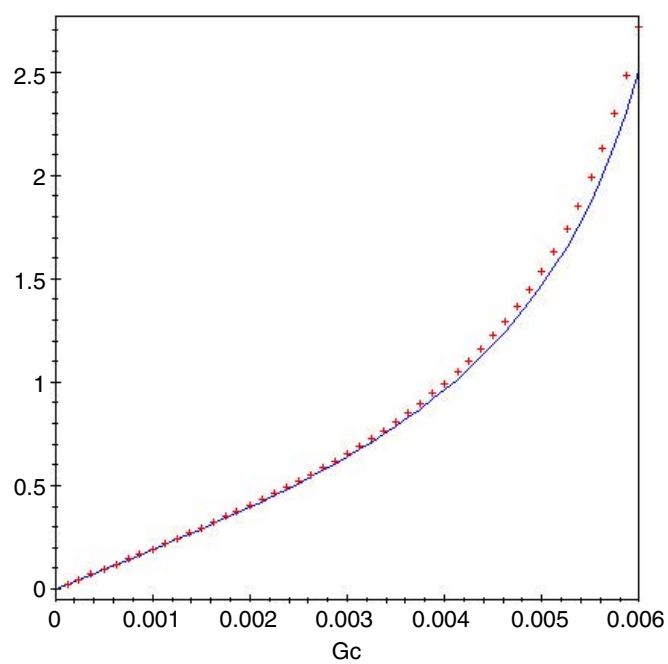

Figure 3. Modal loss factor $\eta_{n}$ as a function of the control gain constant $G_{c}$. Simply supported beam. Study of the first vibration mode. Direct and proportional velocity feedback control. (x) results from formula (39), (continuous line) results from formula (54).

In the case of a damping control, the damping matrix in (50) never has a definite sign. So the dynamic problem (42), (50) is not well posed, whatever the gain. There is a spillover phenomenon in the membrane problem if $G_{c}$ is positive and in the bending problem if $G_{c}$ is negative. Thus, it would be dangerous to solve numerically the complete dynamical problems established in this part, as well as the electromechanical problems of section 2 associated with the presented control laws. Nevertheless, one can find such calculations in the recent literature. Note that the modal analysis of this paper remains consistent, because it is restricted to few modes, while the instabilities and the ill posed problems are related to high eigenfrequencies.

\section{Conclusion}

In this paper, simple models and analytical modal solutions for piezoelastic/elastic/piezoelastic beams have been presented. Two feedback control laws have been considered and constitutive laws of the whole system beam-control device have been established. These global laws can be of elastic type (conservative or not) or of viscoelastic type.

In the case of thin piezoelectric layers, a pure bending model is sufficient to define bending vibration modes. Within this very simple modelling, the behaviour of a system is summarized by a stiffness bending modulus, that depends on the geometrical and material data of the laminate and on the control gain. The latter modulus can be real or complex, according to the type of control law. This means that the control can modify the stiffness of the system or induce some damping. Such a very simplified analysis can be useful to describe more complicated systems such as hybrid passiveactive damping, laminated plates or more intricate control devices.

Furthermore, a complex modal approach has been considered to characterize active damped systems. This method had been widely applied for viscoelastic damping. 
Within this method, each complex mode is associated with a complex eigenvalue and this defines an eigenfrequency and a loss factor per mode. In our opinion, the modal approach should be more extensively used in the case of piezoelectric laminates and active control. It permits one to simply characterized the damping properties of an active system, independently of the applied loads.

\section{Appendix: approximate analysis of the electric problem}

The electric problem in the sensor is studied according to the assumptions described in section 2. It is reduced to a transversal problem, associating the constitutive law (8) and the following ones:

$$
\frac{\partial D_{z}}{\partial z}=0, \quad \phi(r=0)=0, \quad D_{z}(r=1)=0
$$

where the adimensional variable $r$ ( $r=0$ refers to the bottom layer and $r=1$ refers to the top layer) is used. This assumes that the central layer is conductive and that the electric current due to the central system is neglected. The variational form of (A.1) is

$$
\int_{0}^{1} D_{z}(r) \delta \phi^{\prime}(r) \mathrm{d} r=0
$$

for any $\delta \phi(r)$ that is zero for $r=0$. With account of the constitutive law, we get

$$
\int_{0}^{1} \phi^{\prime}(r) \delta \phi^{\prime}(r) \mathrm{d} r=\frac{e_{31}^{*} h_{s}}{\epsilon_{33}^{*}} \int_{0}^{1} \varepsilon_{1}(r) \delta \phi^{\prime}(r) \mathrm{d} r .
$$

Next, we assume that the potential $\phi(r)$ varies in a quadratic manner across the layer and a Galerkin approximation is applied:

$$
\phi(r)=\phi_{1} r+\phi_{2} r(1-r) \quad \delta \phi(r)=r \quad \text { and } \quad \delta \phi=r^{2} .
$$

So equation (A.3) leads to the following approximated system:

$$
\left[\begin{array}{cc}
1 & 0 \\
0 & \frac{1}{3}
\end{array}\right]\left\{\begin{array}{l}
\phi_{1} \\
\phi_{2}
\end{array}\right\}=\frac{e_{31}^{*} h_{s}}{\epsilon_{33}^{*}}\left\{\begin{array}{c}
\int_{0}^{1} \varepsilon_{1} \mathrm{~d} r \\
\int_{0}^{1} \varepsilon_{1}(1-2 r) \mathrm{d} r
\end{array}\right\} .
$$

As $\varepsilon=u^{\prime}-z w^{\prime \prime}$, the system (A.5) has the following solution:

$$
\begin{gathered}
\phi_{1}=\frac{e_{31}^{*} h_{s}}{\epsilon_{33}^{*}}\left(u^{\prime}-z_{s} w^{\prime \prime}\right) \\
\phi_{2}=\frac{e_{31}^{*} h_{s}^{2}}{2 \epsilon_{33}^{*}} w^{\prime \prime} .
\end{gathered}
$$

It must be noted that $\phi_{2}$ is proportional to $h_{s}^{2}$.

To know if the potential $\phi_{2}$ has an important part in the bending stiffness $(E I)_{\mathrm{eq}}$, we look at the contribution of the sensor in the bending moment, $M_{s}$, which is defined by

$$
M_{s}=-\int_{0}^{1} \sigma_{1} z \mathrm{~d} z
$$

$\sigma_{1}$ defined in (2) is rewritten with account of expressions (A.6):

$$
\sigma_{1}=-c_{11}^{*} z w^{\prime \prime}-\frac{e_{31}^{* 2}}{\epsilon_{33}^{*}}\left(z_{s} w^{\prime \prime}-\frac{h_{s}^{2}}{2} w^{\prime \prime}(1-2 r)\right) .
$$

Then, the part of $(E I)_{\mathrm{eq}}$ due to the sensor, denoted $(E I)_{s}$, is equal to

$$
(E I)_{s}=c_{11}^{*} \int_{s} z^{2} \mathrm{~d} z+\frac{e_{31}^{* 2}}{\epsilon_{33}^{*}} z_{s} \int_{s} z \mathrm{~d} z-\frac{e_{31}^{* 2} h_{s}^{2}}{\epsilon_{33}^{*} 2} \int_{s}(1-2 r) z \mathrm{~d} z
$$

where $s$ denotes the sensor. The last term of (A.8), which comes from the quadratic term $\phi_{2}$, is function of $h_{s}^{2}$. That is why it can be neglected for thin skins, as explained in section 2.6.

\section{References}

[1] Benjeddou A, Deü J F and Letombe S 2002 Free vibrations of simply-supported piezoelectric adaptative plate: an exact sandwich formulation Thin-Walled Struct. 40573

[2] Benjeddou A, Trindade M A and Ohayon R 1997 A unified beam finite element model for extension and shear piezoelectric actuation mechanisms J. Intell. Mater. Syst. Struct. 81012

[3] Duigou L, Daya E M and Potier-Ferry M 2003 Iterative algorithms for non-linear eigenvalue problems. Application to vibrations of viscoelastic shells Comput. Methods Appl. Mech. Eng. 1921323

[4] Fernandes A and Pouget J 2002 An accurate modelling of piezoelectric multi-layer plates Eur. J. Mech. A 21629

[5] Galucio A C, Deü J F and Ohayon R 2005 A fractional derivative viscoelastic model for hybrid active-passive damping treatments in time domain-application to sandwich beams J. Intell. Mater. Syst. Struct. 16257

[6] Geradin M and Rixen D 1994 Mechanical Vibrations. Theory and Applications to Structural Dynamics (New York: Wiley)

[7] Gopinathan S V, Varadan V V and Varadan V K 2000 A review and critique of theories for piezoelectric laminates Smart Mater. Struct. 924

[8] Kapuria S, Ahmed A and Dumir P C 2005 An efficient coupled zigzag theory for dynamic analysis of piezoelectric composite and sandwich beams with damping J. Sound Vib. 279345

[9] Liew K M, He X Q and Kitipornchai S 2004 Finite element method for the feedback control of FGM shells in the frequency domain via piezoelectric sensors and actuators Comput. Methods Appl. Mech. Eng. 193257

[10] Maurini C, Pouget J and dell'Isola F 2004 On a model of layered piezoelectric beams including transverse stress effect Int. J. Solids Struct. 414473

[11] Soni M L 1981 Finite element analysis of viscoelastically damped sandwich structures Shock Vib. Bull. 5597

[12] Trindade M A and Benjeddou A 2002 Hybrid active-passive damping treatments using viscoelastic and piezoelectric materials: review and assessment J. Vib. Control 8699

[13] Zhang X D and Sun C T 1996 Formulation of an adaptive sandwich beam Smart Mater. Struct. 5814 